Exploration of the functional conservation across major phylogenic boundaries to define the ancestry of genetic functions will also be important. Within this framework, the study of biological innovations, such as the origin of new genes or their neo- or sub-functionalization, will be of particular relevance to legume biologists, considering the restricted occurrence of nitrogen-fixing symbiosis and its proven functional link to the much older (approximately 400 million years ago) and certainly more ubiquitous association of plants with mycorrhizal fungi $[19,20]$.

The availability of legume genomes opens an unusually valuable treasure chest, where efficient sequestration of nitrogen, phosphate and carbon has been hiding within the dynamic structure of gene networks. Unwinding this biological wealth might provide an important insight on how to preserve nonrenewable resources and the health of the environment at the same time as fulfilling the increasing need for sustainable biomass production.

\section{Acknowledgements}

We thank Alex Molnar for preparing Figure 1. K.S. is supported by grants from Agriculture and Agri-Food Canada and the National Science and Engineering Research Council of Canada (NSERC, grant R3277A01) and J.S. is supported by the Danish National Research Foundation.

\section{References}

1 Mendel, G. (1866) Versuche über Pflanzen-hybriden. Verh. Natforsch. Ver. Brünn 4, 3-47. English translation from web site http:// www.mendelweb.org/Mendel.html

2 Darwin, C.R. (1880) The Power of Movement in Plants, John Murray

3 Sato, S. et al. (2008) Genome structure of the legume, Lotus japonicus. DNA Res. DOI: 10.1093/dnares/dsn008

4 Dolye, J.J. and Luckow, M.A. (2003) The rest of the iceberg. Legume diversity and evolution in a phylogenetic context. Plant Physiol. 131, 900-910

5 Hellriegel, H, and Wilfarth, H. (1888) Untersuchungen über die Stickstoffnahrung der Gramineen und Leguminosen. Beilageheft $z u$ der Zeitschrift des Vereins für Rübenzucker-Industrie des Deutschen Reichs, 234 (see '1888' at http://www.asm.org/membership/ index.asp?bid=16899 for more information)
6 Handberg, K. and Stougaard, J. (1992) Lotus japonicus, an autogamous, diploid legume species for classical and molecular genetics. Plant J. 2, 487-496

7 Barker, D.G. et al. (1990) Medicago truncatula, a model plant for studying the molecular genetics of the Rhizobium-legume symbiosis. Plant Mol. Biol. Rep. 8, 40-49

8 Kaneko, T. et al. (2000) Complete genome structure of the nitrogenfixing symbiotic bacterium Mesorhizobium loti. DNA Res. 7, 331-338

9 Kato, T. et al. (2000) Complete structure of the chloroplast genome of a legume, Lotus japonicus. DNA Res. 7, 323-330

10 Masayoshi, K. (2000) Lotus japonicus 'Miyakojima' MG-20: an earlyflowering accession suitable for indoor handling. J. Plant Res. 113, 507509

11 Szczyglowski, K. et al. (1998) Nodule organogenesis and symbiotic mutants of the model legume Lotus japonicus. Mol. Plant Microbe Interact. 11, 684-697

12 Schauser, L. et al. (1998) Symbiotic mutants deficient in nodule establishment identified after T-DNA transformation of Lotus japonicus. Mol. Gen. Genet. 259, 414-423

13 Perry, J.A. et al. (2003) A TILLING reverse genetics tool and a webaccessible collection of mutants of the legume Lotus japonicus. Plant Physiol. 131, 866-871

14 Colebatch, G. et al. (2004) Global changes in transcription orchestrate metabolic differentiation during symbiotic nitrogen fixation in Lotus japonicus. Plant J. 39, 487-512

15 Nishimura, R. et al. (2002) HAR1 mediates systemic regulation of symbiotic organ development. Nature 420, 426-429

16 Radutoiu, S. et al. (2003) Plant recognition of symbiotic bacteria requires two LysM receptor-like kinases. Nature 2003, 585-592

17 Murray, J.D. et al. (2007) A cytokinin perception mutant colonized by Rhizobium in the absence of nodule organogenesis. Science 315 , 101-104

18 Choi, H-K. et al. (2004) Estimating genome conservation between crop and model legume species. Proc. Natl. Acad. Sci. U. S. A. 101, 1528915294

19 Duc, G. et al. (1989) First report of non-mycorrhizal plant mutants $\left(\mathrm{Myc}^{-}\right.$) obtained in pea (Pisum sativum L.) and fababean (Vicia faba L.). Plant Sci. 60, 215-222

20 Kistner, C. et al. (2005) Seven Lotus japonicus genes required for transcriptional reprogramming of the root during fungal and bacterial symbiosis. Plant Cell 17, 2217-2229

1360-1385/\$ - see front matter. Crown Copyright @ 2008 Published by Elsevier Ltd. All rights reserved.

doi:10.1016/j.tplants.2008.08.001 Available online 30 August 2008

\title{
Boosting tandem affinity purification of plant protein complexes
}

\author{
Jelle Van Leene ${ }^{1}$, Erwin Witters ${ }^{2,3}$, Dirk Inzé ${ }^{1}$ and Geert De Jaeger ${ }^{1}$ \\ ${ }^{1}$ Department of Plant Systems Biology, Flanders Institute for Biotechnology and Department of Molecular Genetics, Ghent \\ University, Technologiepark 927, 9052 Gent, Belgium \\ ${ }^{2}$ Center for Proteome Analysis and Mass Spectrometry, University of Antwerp, Groenenborgerlaan 171, 2020 Antwerp, Belgium \\ ${ }^{3}$ Flemish Institute for Technological Research, VITO, Boeretang 200, 2400 Mol, Belgium
}

\begin{abstract}
Protein-interaction mapping based on the tandem affinity purification (TAP) approach has been successfully established for several systems, such as yeast and mammalian cells. However, relatively few protein complex purifications have been reported for plants. Here, we highlight solutions for the pitfalls and propose a major breakthrough in the quest for a better TAP tag in plants.
\end{abstract}

Corresponding author: De Jaeger, G. (geert.dejaeger@psb.ugent.be).

\section{The rise of TAP}

Over the past 20 years, a wide variety of methods have been developed to explore protein interactions. Co-immunoprecipitation or yeast two-hybrid were often the method of choice, but the emergence of powerful, ultrasensitive high-throughput mass spectrometry (MS), together with the availability of comprehensive protein sequence repertoires, has favored the development of methods relying on in situ affinity purification of protein complexes. 
Especially, the tandem affinity purification (TAP) approach, based on the expression of a bait protein fused to a double affinity tag (the TAP tag), has proven to be of great value. The classical TAP tag consists of two immunoglobulin G (IgG)-binding domains of protein A from Staphylococcus aureus, a specific protease cleavage site for elution by addition of the tobacco (Nicotiana tabacum) etch virus (TEV) protease and a calmodulin-binding peptide (CBP). Purification steps were optimized for highest recovery while maintaining protein complex integrity. TAP of protein complexes was first demonstrated in yeast (Saccharomyces cerevisiae) [1] and was soon applied in a wide variety of organisms, giving rise to high-quality and comprehensive protein-interaction networks [2,3]. Nowadays, databases are filled with protein-interaction data from TAP experiments, but in the plant research field, the TAP approach considerably lags behind. Here, we review and discuss the use of TAP in plants and provide solutions to problems associated with the technology.

\section{TAP in plants: a brief overview}

Until now, only a limited number of purifications from plant material through TAP have been reported, and most have been performed with the traditional yeast tag or with a plant-adapted version, called the improved TAP tag (TAPi) [4]. The latter contains the same modules as the traditional yeast TAP tag but with an optimized codon sequence for plants and an intron for higher gene expression and without cryptic nuclear localization signals. Both the traditional and the TAPi tags were used with success for complex purifications from Arabidopsis thaliana [4-10] and rice (Oryza sativa) [11,12]. A protein complex from Arabidopsis was also purified with an alternative TAP tag (TAPa), in which the CBP domain was replaced by a 9xMyc and $6 x$ His sequence, preventing the non-specific purification of endogenous calmodulin-binding proteins and allowing purification of cation-dependent enzyme complexes because no EGTA-containing buffers are required [13]. Furthermore, the TEV cleavage site was replaced by the more-specific and low-temperature-active human rhinovirus $3 \mathrm{C}$ protease cleavage site. Although this tag has often been used as an epitope tag for protein gel blotting [14] and in co-immunoprecipitation [14,15] or chromatin immunoprecipitation experiments [15], only a single protein complex has been characterized with the TAPa tag [13]. In conclusion, in plants, the traditional yeast TAP and the TAPi tags perform best so far.

\section{TAP in plants: pitfalls and solutions}

Approximately ten years after the proof of concept in yeast, the few TAP data from plants demonstrate that problems are associated with the method. Indeed, unlike in yeast, efficient homologous recombination is not feasible in higher plants. So, the endogenous protein and the tagged counterpart will compete for complex assembly. To overcome this pitfall, different strategies can be followed. The TAP-tagged protein can be introduced into a mutant background, where the endogenous protein is suppressed by RNA interference [16] or is eliminated by transferred DNA (T-DNA) insertion [13]. These complementation approaches determine the functionality of the tagged protein and increase the success rate of the purification because more interactors are available for complex assembly with the tagged protein. A more generic approach for increasing competition is overexpression of the tagged bait, a strategy used in all successful TAP reports in plants so far. Another problem is false negative interactors, especially when low-abundant complexes are studied. Because proteins are present in a high dynamic range, varying from only $10-100$ copies to more than $10^{7}$ copies per cell, and because they cannot be amplified like polynucleotides by PCR, the success rate of TAP depends on the amount of protein complexes purified and the MS sensitivity. One possibility for circumventing the problem of false negatives is the combination of multiple TAP eluates from parallel purifications [13]. Alternatively, the amount of protein extract can be increased before purification. When studying basic cell biological processes, plant cell suspension cultures have a major advantage compared to whole plants because they are fast growing and provide an unlimited supply of synchronizable biological material. Moreover, the PSB-D culture used previously [5] has a ploidy level of $8 \mathrm{C}$, meaning that more proteins are available for complex assembly. The suspension culture is ideal for investigating the cell cycle [5], but it can also be used to isolate complexes involved in other fundamental processes, such as primary metabolism, gene expression or cell-wall synthesis.

\section{The quest for a better TAP tag}

Despite the valuable strategies described above, it was clear that a major leap forward would only be possible through further optimization of complex purification. Therefore, we evaluated different TAP tags for plant cells (Figure 1a). In line with the TAPa tag [13], we replaced the CBP part in the traditional TAP tag with linear peptide epitopes to reduce background. Although the final TAP eluates obtained with this SFZZ tag (Figure 1a) looked often much 'cleaner' on gel, the purified amount of complexes was systematically low and only a few interacting proteins could be sequenced (see supplementary Table S1). Probably the TAPa tag also has to deal with this low complex yield because six different TAP eluates had to be pooled to identify the COP9 signalosome complex [13]. Despite a layer of background proteins sticking to the calmodulin resin, in our laboratory, the best results with respect to complex yield were, until recently, always obtained with the traditional or the TAPi tags [4]. Background proteins sticking to the resins and other false positives from non-specific binding to complexes after protein extract preparation can be determined by mock and exogenous protein purifications using, for example, green fluorescent protein (GFP) or $\beta$-glucuronidase. This list of proteins is then systematically subtracted from the original prey list. To get rid of bait-specific false positives absent in the control TAP list, it is also valuable to repeat purifications and to give more confidence to interactions that were confirmed in multiple experiments [5] or that achieved the best protein identification scores. Assigning confidence scores to interactions by integrating interaction data with other data sources is also rewarding, a method 
(a) Overview of tested TAP tags

TAP

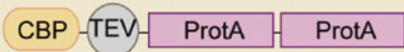

GS tag

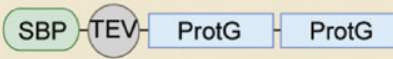

SFZZ

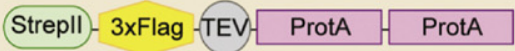

(b) Higher bait expression with GS tag fusions

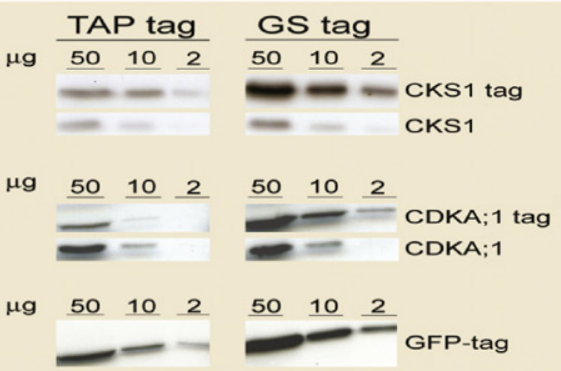

(c) Lower background and higher complex yield with GS tag compared to TAP tag
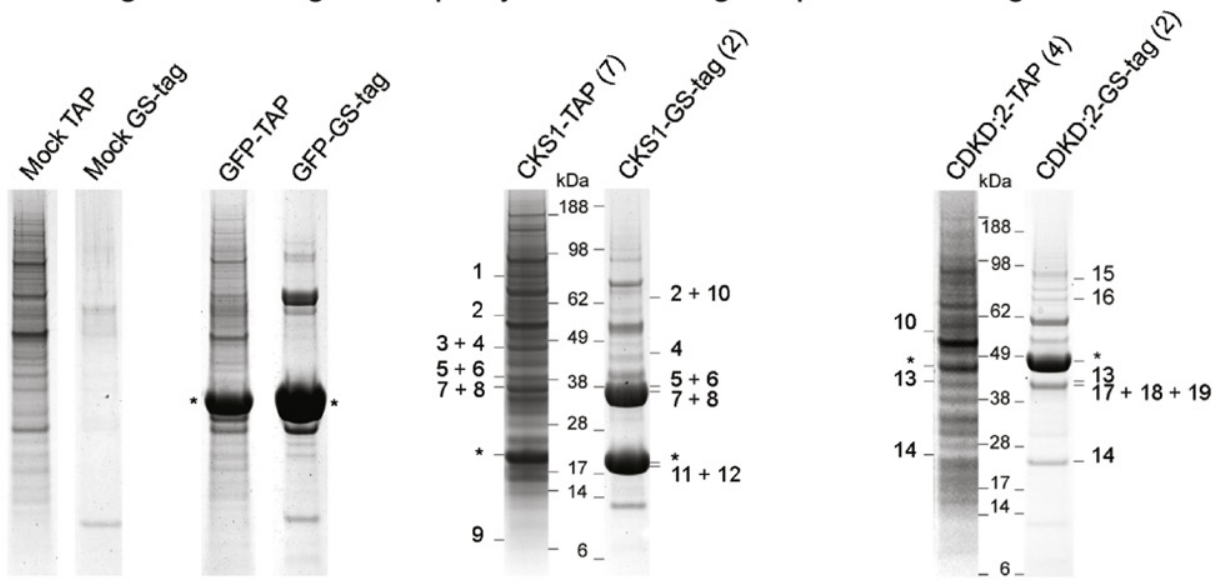

1) AT3G53880 aldo/keto reductase
2) AT4G14310 unknown
3) AT1G47230 CyCA3;4
4) AT2G22490 CycD2;1
5) AT1G76540 CDKB2;1
6) AT1G20930 CDKB2;2
7) AT3G54180 CDKB1;1
8) AT3G48750 CDKA;1
9) Arath05g16630 (Eugene) unknown
10) AT4G16143 importin alpha-2
11) AT5G40460 unknown
12) AT1G10690 unknown
13) AT5G27620 CycH; 1
14) AT4G30820 MAT1
15) AT1G03190 UVH6
16) AT1G55750 TFIIH-related
17) AT1G32380 PRS2
18) AT2G35390 PRS1
19) AT2G44530 PRS, putative

(d) Three fragment recombination for C-terminal GS tag fusions

(e) Two fragment recombination for $\mathrm{N}$-terminal GS tag fusions
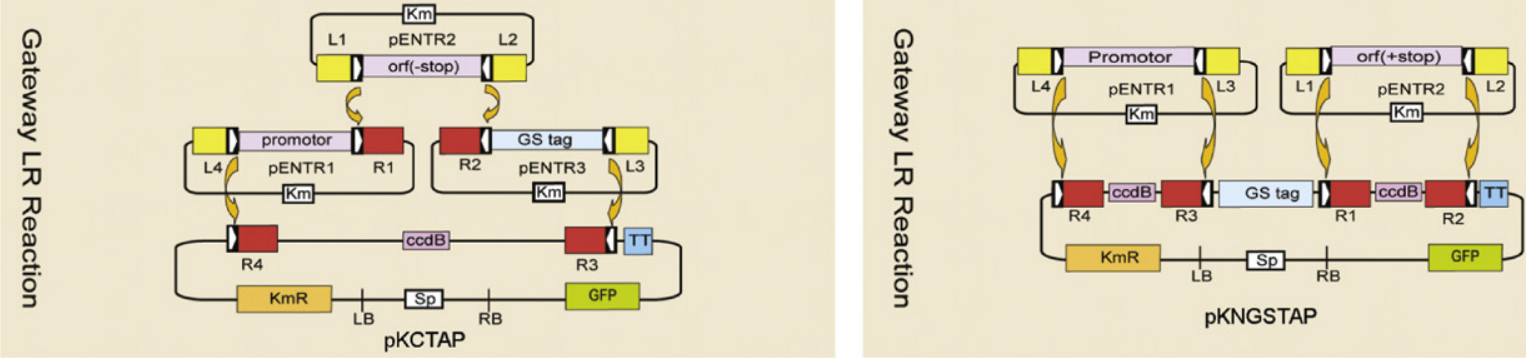

Figure 1. Evaluation of alternative TAP tags in Arabidopsis cell suspension culture. (a) Overview of tested TAP tags. Abbreviations: 3xFlag, three copies of Flag tag; ProtA immunoglobulin G (lgG)-binding domain of protein A; ProtG, IgG-binding domain of protein G; SBP, streptavidin-binding peptide; Strepll, Strepll tag. (b) Higher bait expression with GS tag fusions: $50 \mu \mathrm{g}, 10 \mu \mathrm{g}$ and $2 \mu \mathrm{g}$ of total protein extracts of cultures expressing CKS1-tag, CDKA;1-tag or GFP-tag were separated via SDS-PAGE. Both TAP and GS tag fusions were analyzed via immunoblotting with antibodies against bait proteins. For CKS1 and CDKA; 1 , endogenous protein levels are also shown. (c) Comparison of final eluates with the traditional TAP and GS tag protocols: final eluates were precipitated, separated on 4-12\% NuPAGE gradient gels and visualized with Coomassie G. Background levels were analyzed with mock and GFP tag purifications. Proof of concept was demonstrated for CKS1 (At2g27960) and CDKD;2 (At1g66750). Preys identified via MALDI-TOFTOF and confirmed in multiple experiments are indicated (see supplementary Table S2). The number of purifications used to determine the confirmed interactors is shown in parentheses. Asterisks indicate bait proteins. (d) Schematic representation of the cloning strategy for GS fusions to the C terminus or (e) to the $\mathrm{N}$ terminus of the bait protein. Abbreviations: ccdB, toxic killer gene for negative selection; KmR, neomycin phosphotransferase II gene for selection of transformed plant cells; LB, left border for T-DNA insertion; RB, right border for T-DNA insertion; Sp, streptomycin and spectinomycin resistance gene; TT, cauliflower mosaic virus $35 \mathrm{~S}$ transcription terminator.

often applied in prediction of protein-protein interactions $[17,18]$.

\section{A new TAP tag for plants: the GS tag}

In our continuous search for an ideal TAP tag for plants, we recently evaluated the GS tag [19], which combines two IgG-binding domains of protein $G$ with a streptavidin-binding peptide, separated by two TEV cleavage sites. This tag, developed to study mammalian protein complexes, has been reported to give a tenfold increase in bait recovery compared to the traditional TAP tag. We adapted the GS protocol for plant cells (see online supplementary Box S1) and tested background levels by comparing two mock and two GFP purifications with the traditional TAP tag (Figure 1c). Background levels, counted as the average number of proteins identified in two experimental repeats, dropped from 62 to 8 and from 87 to 11 proteins for mock and GFP purifications, respectively, making MS analysis much less labor intensive and the identification of genuine protein interactions easier, 
especially with low-abundant complexes. An additional benefit of the GS tag is the higher cellular concentration levels of the bait protein (Figure 1b) and the concomitantly higher complex incorporation and yield, as shown by the stronger A-type cyclin-dependent kinase (CDKA;1) band in the CDK subunit 1 (CKS1) GS-tag purification (Figure 1c). As a final proof of the GS tag superiority, we present results obtained with two cell-cycle baits, CKS1 and the D-type CDK-activating kinase CDKD;2 (Figure 1c). Only the experimentally confirmed interactors are represented and compared with those obtained with the traditional TAP tag [5] (see also online supplementary Table S2). For CKS1, most of the interactors confirmed previously in seven purifications with the traditional TAP tag were found with the GS protocol with only two purifications. In addition, some new interesting interactions could be detected with the GS tag only. The known partners of $\mathrm{CDKD} ; 2$, the $\mathrm{H}$-type cyclin $(\mathrm{CycH} ; 1)$ and CDK-activating kinase assembly factor 'ménage à trois' 1 (MAT1), previously discovered in four purifications with the traditional TAP tag, were identified in two purifications with the GS protocol. Moreover, using the GS tag we demonstrated that, as in rice [11], the Arabidopsis CDKD;2 is part of the transcription factor IIH (TFIIH) complex, because ultraviolet hypersensitive 6 (UVH6) and a TFIIH-complex-related transcription factor co-purified. Furthermore, CDKD;2 might link regulation of cell division with nucleotide biosynthesis because of co-purification of three phosphoribosyl diphosphate synthetases (PRSs).

\section{Conclusion and perspectives}

We have shown that the GS tag outperforms the traditional TAP tag in plant cells, both concerning specificity and complex yield. Recently, we replaced the TEV protease cleavage sites in the GS tag with the rhinovirus 3C cleavage site for improved protein complex stability during purification. Combined with the latest and most sensitive MS technology, this tag should bring protein complex analysis in plants to its full bloom. Cloning with these tags is compatible with the Gateway system [20] (Figure 1d,e), and vectors for C- or N-terminal cloning are available at http://www.psb.ugent.be/gateway/.

\section{Acknowledgements}

We thank Giulio Superti-Furga and Tilmann Bürckstümmer for providing the plasmid encoding the GS tag, all members of the Functional Proteomics group at the Plant Systems Biology Department, including Emilie De Witte, and all members of the Centre for Proteomics and Mass Spectrometry at Antwerp University for technical assistance, and Martine De Cock for help in preparing the manuscript. This work was supported by grants from the Institute for the Promotion of Innovation through Science and Technology in Flanders (Generisch Basisonderzoek aan de Universiteiten [GBOU] grant no. 20193) and from the European Commission in the 6th Framework Programme (Agronomics; LSHG-CT-2006-037704).

\section{Supplementary data}

Supplementary data associated with this article can be found, in the online version, at doi:10.1016/j.tplants.2008. 08.002 .

\section{References}

1 Rigaut, G. et al. (1999) A generic protein purification method for protein complex characterization and proteome exploration. Nat. Biotechnol. 17, 1030-1032

2 Gavin, A.C. et al. (2006) Proteome survey reveals modularity of the yeast cell machinery. Nature 440, 631-636

3 Krogan, N.J. et al. (2006) Global landscape of protein complexes in the yeast Saccharomyces cerevisiae. Nature 440, 637-643

4 Rohila, J.S. et al. (2004) Improved tandem affinity purification tag and methods for isolation of protein heterocomplexes from plants. Plant $J$. $38,172-181$

5 Van Leene, J. et al. (2007) A tandem affinity purification-based technology platform to study the cell cycle interactome in Arabidopsis thaliana. Mol. Cell. Proteomics 6, 1226-1238

6 Van Aken, O. et al. (2007) Mitochondrial type-I prohibitins of Arabidopsis thaliana are required for supporting proficient meristem development. Plant J. 52, 850-864

7 Xing, D. and Chen, Z. (2006) Effects of mutations and constitutive overexpression of EDS1 and PAD4 on plant resistance to different types of microbial pathogens. Plant Sci. 171, 251-262

8 Zhao, Q. et al. (2008) Two distinct interacting classes of nuclear envelope-associated coiled-coil proteins are required for the tissuespecific nuclear envelope targeting of Arabidopsis RanGAP. Plant Cell 20, 1639-1651

9 Takahashi, N. et al. (2008) The DNA replication checkpoint aids survival of plants deficient in the novel replisome factor ETG1. EMBO J. 27, 1840-1851

10 Batelli, G. et al. (2007) SOS2 promotes salt tolerance in part by interacting with the vacuolar $\mathrm{H}^{+}$-ATPase and upregulating its transport activity. Mol. Cell. Biol. 27, 7781-7790

11 Rohila, J.S. et al. (2006) Protein-protein interactions of tandem affinity purification-tagged protein kinases in rice. Plant J. 46, 1-13

12 Abe, M. et al. (2008) Identification of dynamin as an interactor of rice GIGANTEA by tandem affinity purification (TAP). Plant Cell Physiol. 49, 420-432

13 Rubio, V. et al. (2005) An alternative tandem affinity purification strategy applied to Arabidopsis protein complex isolation. Plant $J$. $41,767-778$

$14 \mathrm{Kim}, \mathrm{W} . Y$. et al. (2007) ZEITLUPE is a circadian photoreceptor stabilized by GIGANTEA in blue light. Nature 449, 356-360

15 Zentella, R. et al. (2007) Global analysis of della direct targets in early gibberellin signaling in Arabidopsis. Plant Cell 19, 30373057

16 Forler, D. et al. (2003) An efficient protein complex purification method for functional proteomics in higher eukaryotes. Nat. Biotechnol. 21, 8992

17 Geisler-Lee, J. et al. (2007) A predicted interactome for Arabidopsis. Plant Physiol. 145, 317-329

18 Cui, J. et al. (2008) AtPID: Arabidopsis thaliana protein interactome database - an integrative platform for plant systems biology. Nucleic Acids Res. 36, D999-D1008

19 Burckstummer, T. et al. (2006) An efficient tandem affinity purification procedure for interaction proteomics in mammalian cells. Nat. Methods 3, 1013-1019

20 Karimi, M. et al. (2007) Recombinational cloning with plant Gateway vectors. Plant Physiol. 145, 1144-1154

1360-1385/\$ - see front matter. (C) 2008 Elsevier Ltd. All rights reserved. doi:10.1016/j.tplants.2008.08.002 Available online 2 September 2008 